\title{
ARQUEOLOGÍA E IDENTIDAD ÉTNICA: EL CASO DE BOLIVIA
}

\author{
ARCHAEOLOGY AND ETHNIC IDENTITY: \\ THE CASE OF BOLIVIA
}

\author{
José M. Capriles Flores*
}

\begin{abstract}
En Bolivia, el manejo de la arqueología como un medio de fortalecimiento de identidades étnicas, surge como una demanda social con importantes antecedentes. En este artículo, se presenta un análisis del desarrollo histórico de la forma en que los resultados de las investigaciones arqueológicas han sido empleados en el presente. Se observa que en la actualidad, a pesar de una relación cada vez más importante entre arqueólogos y comunidades locales, la "arqueología académica" falla en proporcionar los estímulos adecuados que le posibiliten un vínculo más dinámico con el público general. Para revertir esta realidad, se requiere de soluciones estructurales, que partan desde la base teórica de la praxis arqueológica y permitan generar un diálogo recíproco entre arqueología y público interesado, principalmente las comunidades indígenas, cuyo pasado y patrimonio cultural son estudiados por los proyectos de investigación arqueológica.
\end{abstract}

Palabras clave: Práctica arqueológica, Bolivia, identidad étnica.

In Bolivia, the management of archaeology as a means of empowering ethnic identities, arises as a social demand. This paper presents an historical analysis of the development of how the results of archaeological investigations have been used. Now, and in spite of increasing collaborations between archaeologists and local communities, "academic archaeology" is failing to provide the appropriate stimulus to facilitate a more dynamic interaction with the general public. To reverse this reality, structural solutions are required, but based on solid theoretical foundations and archaeological practice. This should allow for a productive dialogue between archaeologists and the public, in particular the indigenous communities whose past and cultural patrimony is studied by archaeological projects.

Key words: Archaeological practice, Bolivia, ethnic identity.

En el presente ensayo, trataré de realizar una revisión sintética del desarrollo del pensamiento arqueológico en Bolivia, en relación al manejo en el presente de la información derivada de la investigación arqueológica. Considerando que la arqueología en cuanto a ciencia social debe responder a las demandas de la sociedad, se analiza su aporte en relación al fortalecimiento de la identidad étnica de los grupos nativos cuyo pasado investiga. Se rescata la importancia de los pocos aportes teóricos enfocados en la difusión de los datos arqueológicos a un público más amplio. Sin embargo, se propone que la articulación entre la información recuperada de la investigación arqueológica y la sociedad en su conjunto, debe plantearse a través de políticas dirigidas al fortalecimiento de las identidades étnicas de los grupos del presente (Capriles 2001). En este artículo, siguiendo la propuesta de Jones (1997), utilizaremos el concepto de identidad étnica como "ese aspecto de la auto- conceptualización de un individuo que resulta de su identificación con un grupo más amplio en oposición a otros sobre la base de una diferenciación cultural percibida y/o descendencia común (Jones 1997:xiii, traducción del autor). En este sentido, como se verá más adelante, consideramos el pasado como uno de los elementos fundamentales para la estructuración social de la identidad étnica (Friedman 1992).

\section{El Presente en el Desarrollo Teórico de la Arqueología en Bolivia}

En nuestro país, la arqueología ha tenido un desarrollo bastante lento en comparación a otros países latinoamericanos, alcanzando su institucionalización recién en 1975 (Ponce Sanginés 1995). De ahí que estudios como las descripciones de sitios arqueológicos, reconstrucción de historias culturales y la investigación concentrada en sitios

* Carrera de Arqueología, Universidad Mayor de San Andrés, P.O. Box 499, La Paz, Bolivia. eliflor@ ceibo.entelnet.bo 
monumentales (especialmente Tiwanaku) hayan sido los principales focos de su interés (e.j., Ponce Sanginés 1972). Sin embargo, el proceso de institucionalización de la arqueología boliviana ya había sido iniciado en la década de 1950, influido decididamente por el pensamiento político nacionalista de la época (p.e. la Revolución Nacional de 1952), que fue fortalecido e inclusive legitimado a través del Tiwanaku-centrismo que caracterizó a la que posteriormente se conocería como la Arqueología Nacionalista Boliviana. Como es característico de las arqueologías nacionalistas (Trigger 1984) en Bolivia esta manifestación, se desarrolló a través de la centralización y monopolización de la investigación arqueológica, con una "política científica" orientada hacia el estudio de las raíces prehispánicas de la nacionalidad boliviana, entendida simplemente como la expansión del "Imperio de Tiwanaku". Más aún, la difusión de las nuevas ideas de la Revolución se realizó a través de la centralización y monopolización de las publicaciones arqueológicas y principalmente de los programas educativos, los cuales incluyeron esta nueva visión del pasado prehispánico, con todos sus sesgos ideológicos y políticos.

Durante la década de 1980, ante la crisis del nacionalismo boliviano, varias críticas se desarrollaron en contra de la tradicional Arqueología Nacionalista. Entre ellas, es destacable la propuesta de la creación de una escuela indígena de investigación arqueológica, planteada inicialmente por Rivera Cusicanqui (1980) y luego apoyada por Mamani (1989), quienes condenaron la Arqueología Nacionalista por reivindicar a la nación como pueblo mestizo, legitimando de este modo el colonialismo y negando a los pueblos indígenas el derecho de conocer su pasado. Plantearon en este sentido, una arqueología orientada hacia las necesidades de los pueblos originarios (considerando que en Bolivia la mayoría de la población es indígena), enfocada en la recuperación de valores culturales y en la legitimación de su pasado étnico, empleando para ello principalmente la historia oral (Mamani 1989; Rivera Cusicanqui 1980, 1987).

Una posición diferente, pero concordante en algunos puntos, ofrecía la Arqueología Social Latinoamericana, según la cual, los trabajos arqueológicos, deberían orientarse en beneficio de las poblaciones locales, tanto en la recuperación de la tecnología precolombina como en el fortalecimiento de las identidades nacionales (Lumbreras 1981;
Patterson 1994). De esta manera, la arqueología como ciencia social, tenía el deber de asignarle al pasado una función histórica para el fortalecimiento de las identidades en el presente (Carpio 1997). La influencia de esta escuela de pensamiento en Bolivia no ha sido debidamente analizada, aunque sus efectos no fueron tan importantes como en otros países (Patterson 1994).

Por otra parte, paralelamente a la decadencia de la Arqueología Nacionalista, una serie de proyectos arqueológicos principalmente norteamericanos empezaron a ejecutarse en Bolivia. Su influencia en nuestro país (al igual que en otros países vecinos), fue decisiva para el desarrollo de las escuelas locales de pensamiento (así como de la práctica de la arqueología en sí misma). Esto es particularmente cierto considerando que, recién en 1984 se crea la Carrera de Arqueología en la Facultad de Ciencias Sociales de la Universidad Mayor de San Andrés en La Paz, generando de esta manera una provechosa dinámica entre docentes y estudiantes bolivianos y norteamericanos.

A nivel teórico, la dominante Arqueología Procesualista norteamericana e inglesa, a través de la investigación sistemática y científica del registro arqueológico, tenía como objetivo principal llegar a inferir los procesos de cambio cultural y la dinámica del comportamiento humano en forma de leyes generales (Watson et al. 1974). La Nueva Arqueología, como también se conoce a esta posición teórica, mediante "[1]a aplicación rigurosa de un enfoque positivista tenía el efecto de eliminar los elementos subjetivos y de establecer una base para la interpretación científica objetiva de los datos arqueológicos" (Trigger 1992:282).

Con este marco de referencia, la Arqueología Procesual descartó varios de los aspectos más importantes del análisis sociocultural, por ejemplo, el carácter esencialmente significativo de la cultura material, el papel que desempeñan los individuos en la generación del cambio y la variación y la importancia del simbolismo y la ideología en los procesos de estructuración social, entre muchos otros (Hodder 1988). Pero, además, dejó de lado las otras lecturas del pasado, considerándolas alternativamente como especulaciones o mitos.

Es así que a partir de la década de 1980, la Arqueología Procesualista empezó a desarrollar un fuerte enfoque autocrítico y varias corrientes de pensamiento alternativo comenzaron a surgir con mucha fuerza bajo el común denominador de la 
corriente post-procesual (Hodder 1988). Algunas de estas propuestas, como la Arqueología Indigenista pero también la Arqueología Marxista, empezaron a alertar sobre: (1) los sesgos que genera en la visión del pasado, el contexto sociocultural que rodea al investigador; y (2) la importancia del pasado en el proceso de construcción de las identidades en el presente (cf. Hodder 1988; Trigger 1992).

Por otro lado, si bien la arqueología en Norteamérica ha sido tradicionalmente una subdisciplina de la antropología, no adquirió un estatus equilibrado con ésta sino hasta los inicios de la década de 1960 (Cowgill 1993; Trigger 1992). Esto permitió que una cantidad cada vez más importante de teorías elaboradas por la antropología, sean también tratadas por la arqueología. No obstante, fue con la crisis del procesualismo durante la década de 1980 que problemáticas que la antropología estaba desarrollando contemporáneamente, recién empezaron a investigarse seriamente (Hodder 1988; Jones 1997). Entre ellas, la importancia de las ciencias sociales en el fortalecimiento de las identidades sociales y étnicas del presente y la búsqueda de los mecanismos que permitan una apropiada integración entre la homogenizante sociedad occidental y la diversidad de sociedades no occidentales.

Recientemente, Albarracin-Jordan (1996, 1997, 1999), ha criticado a la arqueología norteamericana, así como la Arqueología Nacionalista Boliviana y la Arqueología Social Latinoamericana a partir de un marco teórico crítico, analizando principalmente los enfoques del pasado acerca de cómo se ha manejado la arqueología y el pensamiento del pasado en Bolivia y particularmente de Tiwanaku. Concluye que la ideología científica de los actuales proyectos arqueológicos extranjeros en Bolivia responde a criterios políticos neo-colonialistas (Albarracin-Jordan 1997).

Siguiendo esta línea de razonamiento, se puede concluir que a pesar del importante desarrollo teórico experimentado por la arqueología anglosajona a partir de la década de 1980 , específicamente vinculado a la relación entre la disciplina arqueológica y los grupos étnicos del presente, los proyectos de investigación arqueológica norteamericanos en Bolivia han sido lentos a la hora de tomarlo en cuenta, siendo los resultados hasta el momento sumamente limitados. Más aún, considerando que estos proyectos constituyen la mayor parte de la investigación arqueológica ejecutada en Bolivia y su paradigma director, los investigadores nacionales tampoco han tomado en cuenta seriamente la relevancia de la problemática de arqueología y los grupos indígenas en Bolivia.

\section{La Arqueología Como Forma de Reivindicación Étnica}

Hasta este punto, llama la atención que una de las principales problemáticas vinculadas a la arqueología, radica en cómo integrar a la investigación científica (al igual que a la preservación del patrimonio cultural) el proceso de construcción de las identidades étnicas en el presente (Capriles 2001). Varios de los enfoques descritos anteriormente coinciden en que el trabajo del arqueólogo tiene que responder a un grupo determinado de personas, para quienes la investigación arqueológica podrá actuar directamente sobre su identidad, considerando que en última instancia es su pasado el que está siendo estudiado.

En nuestro país, este enfoque de la arqueología no se ha desarrollado adecuadamente. Ya hemos visto las críticas desarrolladas a la Arqueología Nacionalista, cuyos resultados en este campo han sido totalmente insatisfactorios. En Bolivia, son escasos los trabajos que han tomado a la arqueología como un medio para reactivar o fortalecer identidades étnicas de pueblos originarios. Trabajos como los de Portugal Loayza (1997, 2000) o Avilés (1997), más que utilizar la arqueología para reivindicar la identidad étnica de las comunidades originarias, analizan cuál es la función que desempeñan los sitios arqueológicos o más precisamente las ruinas en la construcción de la identidad de los grupos étnicos del presente.

Por otra parte, los resultados de las investigaciones de Albarracin-Jordan (1996, 1999), demostrando la continuidad cultural de la población habitante del valle de Tiwanaku, desmienten una serie de teorías que proponían la destrucción del Imperio de Tiwanaku como producto de una oleada de migraciones aymaras, y apoyan decididamente el fortalecimiento de una profunda identidad étnica de los actuales habitantes de esa región.

A pesar que los trabajos de este tipo no son numerosos, existe una conciencia cada vez mayor por parte de los investigadores que sus resultados pueden incidir, y de hecho inciden, en el desarrollo cotidiano de las comunidades originarias. En la 
actualidad, al menos se puede destacar que existe una mayor interacción entre los proyectos de investigación y las comunidades originarias.

Sin embargo, es alarmante que ante la considerable cantidad y diversidad de movimientos sociales de reivindicaciones étnicas observados en Bolivia durante las últimas dos décadas, ninguno de éstos haya empleado resultados derivados de investigaciones arqueológicas para legitimar su pasado. Para ilustrar claramente este hecho, cabe citar el Manifiesto de Jach'ak' achi, promulgado en abril del 2001. Este documento, a pesar de que incluye una minuciosa reseña histórica y extensos detalles sobre algunos momentos trascendentales de la "Nación Aymara", la parte prehispánica es vagamente desarrollada y se hace más referencia al origen "milenario" y la cosmovisión aymara, que a datos específicos de la historia cultural de este grupo étnico.

Más allá de las connotaciones ideológicas que puede tener el resaltar este hecho, resulta útil como un adecuado indicador para mostrar que el acceso y manejo que el público en general tiene y hace de los conocimientos derivados de la arqueología en Bolivia son prácticamente nulos.

Considero que parte del problema, se deriva del mal manejo que se ha realizado de los resultados de las investigaciones arqueológicas contemporáneas en los programas oficiales de educación pública, los que todavía se reproducen las viejas interpretaciones desarrolladas por la Arqueología Nacionalista, así como su desgastada ideología. Por otra parte, la "cientificidad" (que comprende además una academia, que es el principal [por no decir único] espacio o foro de discusión) y la "objetividad" que ofreció la Nueva Arqueología Norteamericana, han llevado a numerosos investigadores a dejar de lado el carácter relativo y subjetivo que de hecho tiene toda interpretación del pasado, y por tanto a tomar una posición menos consciente con su realidad y el contexto sociocultural en que se insertan sus inferencias.

\section{Articulando: La Arqueología y el Fortalecimiento de las Identidades Étnicas}

Numerosos trabajos antropológicos han demostrado que el pasado es uno de los elementos fundamentales en los procesos de definición de la identidad étnica de un grupo étnico determinado (Friedman 1992; Jones 1997). Al igual que los otros aspectos que caracterizan y conforman la identidad étnica (e.g. Barth 1976) las lecturas del pasado son constantemente modificadas, manipuladas y adecuadas dinámicamente por los actores sociales. No obstante, el pasado continúa configurando uno de los aspectos centrales para la autodefinición consciente y la reproducción social de la identidad étnica. Por ejemplo, uno de los argumentos fundamentales en que se basa la legitimación territorial de los grupos étnicos es su vinculación histórica con el espacio en el que habitan (e.j. Platt 1982). Igualmente, la descendencia y la herencia configuran uno de los caracteres más importantes en los procesos de estructuración social.

Los grupos étnicos en Bolivia, durante los últimos años, se han enfrentado a uno de los procesos de aculturación y asimilación cultural más repentinos e intensivos que han presenciado en toda su historia. Entre los factores causales de este hecho, se puede mencionar la vinculación cada vez más directa entre el área rural y la ciudad, la crisis económica y la necesidad de migrar hacia los centros más poblados, la presencia de nuevos medios de comunicación y la rápida difusión de determinados estereotipos a través de éstos, además de un largo etcétera. Sin embargo, estos mismos grupos étnicos a través de su decisión consciente de resistir a este proceso han generado diversas estrategias para fortalecer su cultura y sobretodo su identidad étnica.

En este sentido, las principales estrategias empleadas por los grupos étnicos para fortalecer su propia identidad a partir del pasado, se fundamentan en la recuperación de la tradición oral (p. ej. Mamani 1989; Rivera Cusicanqui 1980, 1987). A menudo esto se ha realizado a través de la recopilación y publicación de cuentos, mitos y otros derivados de historias orales, así como su introducción en los programas de enseñanza educativa escolar. No obstante, es claro que las nuevas generaciones notan la diferencia entre la percepción del pasado como es representado a través de la tradición oral y otras lecturas de éste, especialmente la historia institucionalizada que es enseñada en los colegios; es decir, la historia como es percibida occidentalmente, esto es, la narrativa de una sucesión cronológica de hechos y/o acontecimientos relevantes, registrados en documentos escritos que demuestran la veracidad de éstos.

La ausencia de una vinculación armónica que pueda reconciliar estas dos, por lo general, contra- 
rias lecturas del pasado, ha generado una importante crisis -aunque todavía no asumida del todocuyas consecuencias son diversas. En relación a la identidad étnica, pueden resumirse en la pérdida del referente histórico de numerosos grupos étnicos y el aceleramiento de su progresiva asimilación cultural hacia el modelo occidental.

Cabe aclarar, que el hecho de que una de estas narrativas sea oral y la otra escrita, no es la razón principal para la consecuencia anteriormente descrita. Después de todo, los numerosos trabajos de recuperación de la tradición oral y la inmensa cantidad de publicaciones con estas características producidas durante los últimos años negarían esta posibilidad (p.e. Paredes Jáuregui 1999). Es, pues, la contradicción entre estos dos discursos y la necesidad de asumir a uno como verdadero y por tanto al otro como falso, la razón fundamental de la crisis. La enseñanza escolar claramente tiende a identificar la historia occidental como verdadera y real, considerando los mitos y cuentos orales como fantasía o distorsiones de la realidad. Este hecho también puede observarse en el énfasis literario que se le ha dado a la tradición oral (p. ej. Paredes Jáuregui 1999), siendo que ésta en realidad trasciende tal esfera y uno de sus aportes principales radica precisamente en la recreación y representación de la historia a través de la memoria colectiva. Sin embargo, debido al contexto no occidental en que está inserta, su narración del pasado es por definición distinta a la narración occidental.

Este aspecto se agudiza mucho más cuando no existe una lectura a partir de la narrativa occidental sobre el pasado prehispánico, o peor aún-como hemos visto anteriormente- cuando la lectura de ésta, está fuertemente sesgada por intereses políticos. Esto último tiene como consecuencia adicional la pérdida de la identidad étnica debido a que se considera al pasado de numerosos grupos étnicos simplemente como inexistente.

Por otro lado, debemos tener en claro que existen numerosas formas en que la arqueología puede contribuir a la sociedad actual y a los grupos étnicos en el presente. El fortalecimiento de la identidad de los grupos étnicos es solamente uno de los aspectos en el cual pueden contribuir sus resultados. En todo caso, debido a que no existen muchas aproximaciones de este tipo y las experiencias ensayadas son aún escasas, entonces tampoco se debe asumir que la forma que propone este trabajo sea la única manera en que puede hacerlo. Por el con- trario, consideramos que existen innumerables formas en que la arqueología puede contribuir al fortalecimiento de identidades étnicas en el presente.

Los trabajos a través de la arqueología experimental y aplicada de tecnologías prehispánicas (ver Michel [1997]) son fundamentales para un análisis reflexivo y crítico de los resultados de estos proyectos con relación a los campos elevados de cultivo. Pero también con la puesta en valor de sitios arqueológicos a través de proyectos de conservación y restauración, al igual que la construcción de museos locales y regionales. Este tipo de iniciativas son positivas en la medida en que generan circuitos económicos que pueden favorecer a las comunidades locales, generando además un espíritu de revalorización cultural dentro de éstas. Sin embargo, numerosas experiencias muestran que no existe una adecuada gestión; el impacto del turismo puede ocasionar consecuencias negativas sobre las regiones y las comunidades locales, entre las cuales la pérdida de la identidad étnica es una de las más importantes. De ahí que la coordinación constante y el diálogo horizontal (Lima, en este volumen) entre las comunidades y los proyectos tanto de investigación como de desarrollo, deben ser los que primen en todas sus etapas de desarrollo.

Ahora bien, en este trabajo proponemos que una de las contribuciones más importantes que puede realizar la arqueología al fortalecimiento de identidades étnicas, es a través de la divulgación de los resultados de sus investigaciones más recientes. De este modo, se podrá popularizar un conocimiento más amplio de la dinámica del pasado prehispánico, que en algunos casos permitirá tener una visión más precisa de la historia cultural prehispánica de las diversas regiones que estudia, y del pasado de numerosos grupos étnicos del presente. Así como la posibilidad de asumir como verdaderas o valederas muchas de las proposiciones de la tradición oral o, por lo menos, plantear la ausencia de información suficiente como para efectivamente descartarlas.

\section{Conclusiones: Perspectivas de la Arqueología como Identidad en Bolivia}

Como hemos visto, es posible hallar varios y distintos enfoques, aproximaciones y perspectivas sobre el manejo de los datos arqueológicos en el presente. En el caso de Bolivia, la investigación 
arqueológica debe trascender las esferas académicas para divulgarse a un público más amplio y generar de esta manera un flujo positivo de información, para de una vez mostrar la utilidad pragmática que tanto se reclama a la arqueología boliviana.

La identidad étnica es un proceso que se constituye y se construye históricamente, por lo que la lectura del pasado es un aspecto fundamental en su generación (Friedman 1992). La contribución de la arqueología en Bolivia a los procesos de reivindicación étnica no ha sido hasta el momento significativa y está lejos de ser satisfactoria. Actualmente, en Bolivia la arqueología no está contribuyendo de manera conveniente a la divulgación de los conocimientos que se han alcanzado tras largos años de trabajo, y tampoco, a la retroalimentación de información a una audiencia más amplia, que desborde su reducido círculo académico. Por tanto, existe la necesidad de desarrollar políticas de acción, que permitan a la población en su conjunto acceder bajo adecuados programas de divulgación a los resultados de las recientes investigaciones y discusiones arqueológicas. Esto simplemente implica, que la arqueología debe asumir su responsabilidad como disciplina científica o, más aún, como ciencia social. De continuar la actual situación, se está cometiendo un acto de injusticia con los grupos étnicos del presente, de quienes todavía se continúa investigando su patrimonio cultural. Es entonces necesario, a través de una integración apropiada con estos grupos, exponerles -e inclusive devolverles- su pasado, desde luego, mediante una forma tan dinámica y suya como el presente y por supuesto el futuro.

De esta manera, se podrá generar una articulación más fluida entre la concepción mítica del pasado y la dinámica que muestran los resultados de las investigaciones arqueológicas más recientes
(Capriles 2001). Pero fundamentalmente se dispondrá de una nueva lectura del pasado de los grupos étnicos del presente, que pueda serles útil ahora y aprendida por las nuevas generaciones. Esta nueva lectura idealmente tomará en cuenta la riqueza de la tradición oral y de la materialidad del registro arqueológico, sin desmarcarse de la narrativa occidental. Así, esperamos coadyuvar en el objetivo general de conservar lo que consideramos como el aspecto más importante y urgente para la preservación de la identidad étnica de estos grupos: la comprobación del referente histórico, tangible y material de su pasado.

Agradecimientos: Quisiera agradecer muy especialmente el apoyo y la paciencia que tuvieron conmigo en innumerables conversaciones Juan Albarracin-Jordan, Dante Angelo, Carlos Lémuz, Pilar Lima, Marcos Michel, Eduardo Pareja y Claudia Rivera Casanovas, quienes contribuyeron de manera significativa al desarrollo de la mayoría de las ideas expuestas en este artículo. También merecen mi más profundo reconocimiento Alejandro Barrientos, Maya Benavides, Rubén Darío Chambi, Alejandra Domic, María Soledad Fernández, Carlos Revilla y Raúl Rodríguez, quienes enriquecieron sobremanera este trabajo con sus entusiastas comentarios y provechosas discusiones en favor de una Antropología y una Arqueología en Bolivia cada vez más reflexivas y autocríticas. Igualmente, quisiera agradecer a Álvaro Romero Guevara y Patricia Ayala Rocabado por su apoyo en la edición final. Finalmente, mis agradecimientos van nuevamente para Patricia Ayala Rocabado por su invitación a participar en el "Encuentro sobre Patrimonio Cultural, Comunidades Indígenas y Arqueología", así como a las comunidades de Ollagüe y del Norte Grande de Chile por la organización de este importante evento.

\section{Referencias Citadas}

\footnotetext{
Albarracin-Jordan, J.

1996 Tiwanaku: Arqueología Regional y Dinámica Segmentaria. Plural Editores, La Paz.

1997 Ciencia, arqueología y patrimonio. Humana 1(1): 41-50.

1999 Arqueología de Tiwanaku: Historia de una Antigua Civilización Andina. Fundación Bartolomé de las Casas, La Paz.
}

Avilés, S. V.

1997 Arqueología y sociedad en Bolivia: Apuntes para un análisis. Textos Antropológicos 8:145-158.

Barth, F.

1976 Introducción. En Los Grupos Étnicos y sus Fronteras: La Organización Social de las Diferencias Culturales, compilado por F. Barth, pp. 9-49. Fondo de Cultura Económica, México D.F. 
Capriles, J.M.

2001 ¿Etnicidad en la Arqueología Boliviana? Una Revisión Crítica. Ponencia presentada en Segundo Simposio de Actualización en Arqueología Boliviana, La Paz.

Carpio, E.H.

1997 Arqueología e identidad nacional. Humana 1(1): 37-40.

Cowgill, G.L.

1993 Distinguished lecture in archaeology: Beyond criticizing new archaeology. American Anthropologist 95: 551-573.

Friedman, J.

1992 The past in the future: History and the politics of identity. American Anthropologist 94:837-859.

Hodder, I.

1988 Interpretación en Arqueología: Corrientes actuales. Editorial Crítica, Barcelona.

Jones, $\mathrm{S}$.

1997 The Archaeology of Ethnicity: Constructing Identities in the Past and Present. Routledge, Londres.

Lumbreras, L.G.

1981 La Arqueología como Ciencia Social. Ediciones PEISA, Lima.

Mamani, C.

1989 History and prehistory in Bolivia: What about the indians? En Conflict in the Archaeology of Living Traditions, editado por R. Layton, pp. 46-59. Unwin Hyman, Londres.

Michel, M.R.

1997 Campos elevados en el altiplano peruano-boliviano y las llanuras benianas: Tecnología del pasado que retorna al presente. Humana 1(1):51-64.
Paredes Jáuregui, N.T.

1999 El cuento oral. Correveydile Revista Boliviana de Cuento 11:5-13.

Patterson, T.C.

1994 Social archaeology in Latin America: An appreciation. American Antiquity 59:531-537.

Platt, T.

1982 Estado Boliviano y Ayllu Andino. Tierra y Tributo en el Norte de Potosí. Instituto de Estudios Peruanos, Lima.

Ponce Sanginés, C.

1972 Tiwanaku: Espacio Tiempo y Cultura. Ensayo de Síntesis Arqueológica. Academia Nacional de Ciencias de Bolivia, La Paz.

1995 Tiwanaku: Doscientos Años de Investigaciones Arqueológicas. Producciones CIMA, La Paz.

Portugal Loayza, J.

1997 Un ayllu Uru en el noreste del lago Titicaca Ojchi (Primera parte). Textos Antropológicos 8:11-46.

2000 Un ayllu Uru en el noreste del lago Titicaca: Ojchi (Segunda parte). Textos Antropológicos 11:13-50.

Rivera Cusicanqui, S.

1980 La antropología y arqueología en Bolivia: Límites y perspectivas. América Indígena 40:217-224.

1987 El potencial epistemológico y teórico de la historia oral: De la lógica instrumental a la descolonización de la historia. Temas Sociales 11:49-64.

Trigger, B.G.

1984 Alternative archaeologies: Nationalist, colonialist, imperialist. Man 19:355-370.

1992 Historia del Pensamiento Arqueológico. Editorial Crítica, Barcelona.

Watson, P. J., S.A. LeBlanc y C. Redman 1974 El Método Científico en Arqueología. Alianza Editores, Madrid. 
